\title{
Gross hypertrophic pulmonary osteoarthropathy in a 7-year-old child
}

\author{
NAOMI BARCLAY, M. OGBEIDE, and I. A. GRILLO \\ Departments of Paediatrics and Surgery, University College Hospital, Ibadan, Nigeria
}

\begin{abstract}
Hypertrophic pulmonary osteoarthropathy in children is rare and usually associated with an underlying condition. A case of hypertrophic pulmonary osteoarthropathy secondary to a chronic post-measles lung abscess is presented. There was good radiological evidence of an advanced degree of periosteal reaction. This became much more obvious during an exacerbation of the illness. Films taken 10 months after resection of the affected lobe showed almost complete resolution of the bone condition. A feature that is well known in adults, but has not previously been reported in a child, was the exquisite tenderness of the affected bones. The literature is reviewed.
\end{abstract}

Clubbing in association with empyema was first described by Hippocrates. However, it was not until Bamberger in 1889 and Marie in 1890 described the syndrome of clubbing, bone and joint lesions, and their association with intrathoracic inflammatory conditions, that the phrase hypertrophic pulmonary osteoarthropathy was coined.
In the 80 years since then there have been only 39 paediatric cases fully documented and reported.

In childhood, hypertrophic osteoarthropathy is practically always secondary to an underlying condition (Cavanaugh and Holman, 1965), and the familial variety, sometimes called pachydermoperiostitis, does not present until puberty.

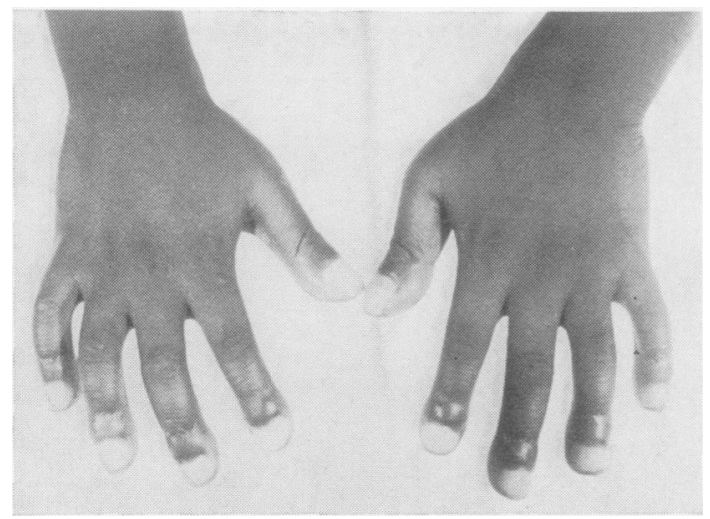

FIG. 1

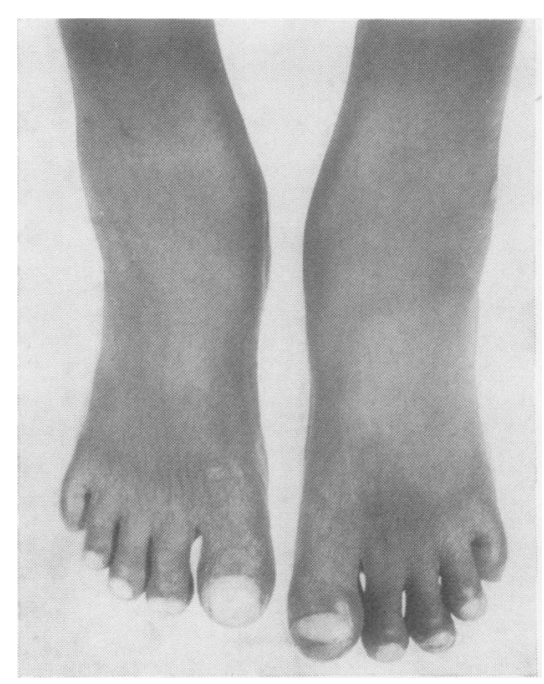

FIG. 2

FIGS 1 and 2. Gross clubbing of the fingers and toes at the time of presentation. Note the swelling of both ankles. 


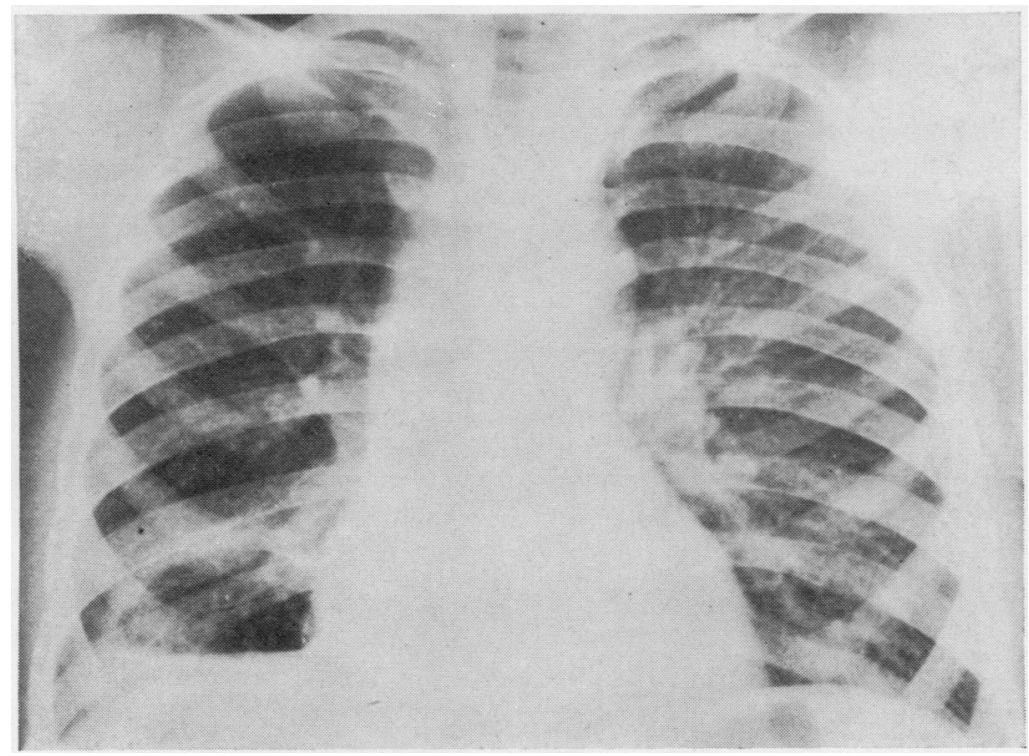

FIG. 3.

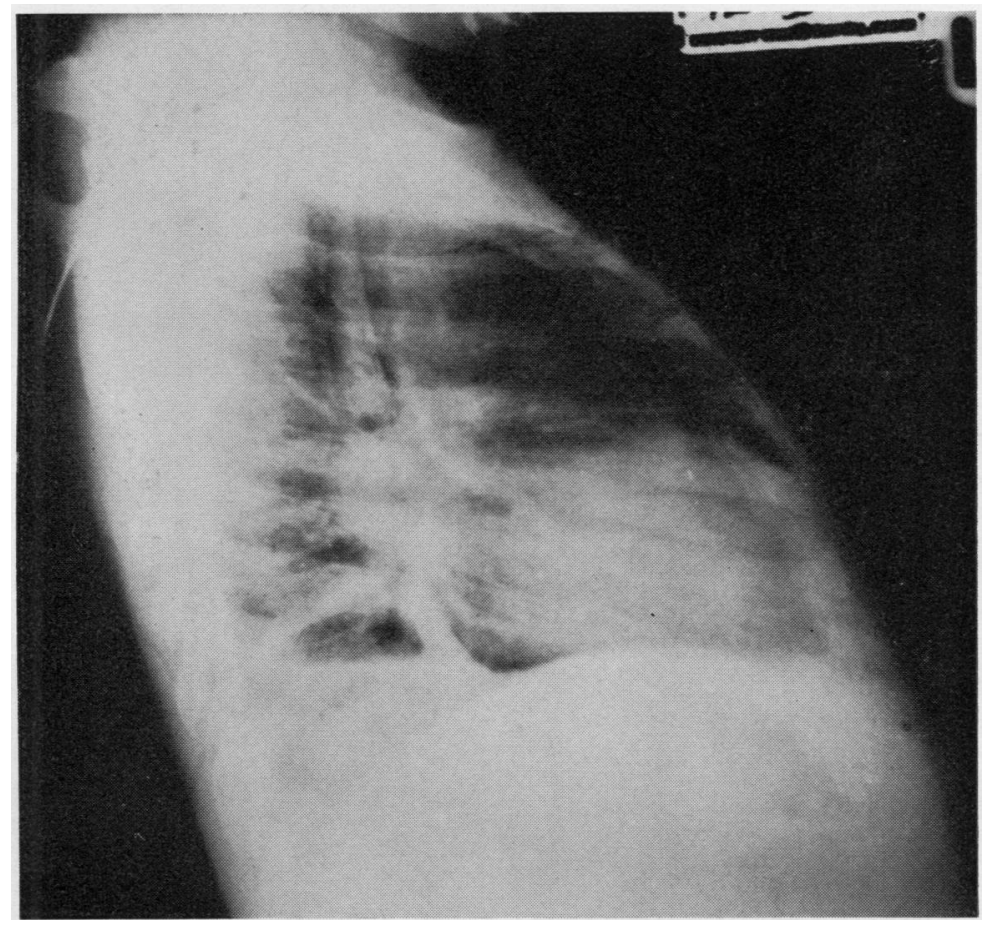

FIG. 4.

FIGS 3 and 4. Chest radiographs showing the abscess cavity in the right lower lobe. 


\section{CASE REPORT}

A 7-year-old Nigerian boy presented at this hospital on 22 October 1969 with a three-year history of productive cough and foul breath. He was the first-born child of his mother. Both parents and sibs were alive and well, with no history of illness or other abnormality.

On further questioning, it was found that the child had had a severe cough following an attack of measles at the age of 18 months, but that this had cleared up. The presenting cough started one year later, in April 1963, and had continued ever since. At the time he was first seen he was bringing up blood-spotted phlegm, but there was no frank haemoptysis. His mother was quite happy about his general health, but on close questioning admitted that he could not fight well with his younger brother. Apart from convulsions on one occasion around 2 years of age, there was nothing else abnormal in the history. There was no history of bone, joint, or chest pain.

Examination at this first visit showed him to be of average development for his age and environment, and to be withdrawn but not acutely ill. No abnormalities were found on clinical examination of the nose, throat, or chest, except that his breath was extremely foul smelling. There was grade IV clubbines of all the fingers and toes (Figs 1 and 2). The ankles were swollen but there was no pitting nor tendernesह on pressure (Fig. 2). There was no cyanosis nor pallo of the mucosae. The spleen and liver were not pal? pable. Chest radiographs showed an abscess cavity? $4 \mathrm{~cm}$. in diameter, in the posterior basal segment of the right lower lobe, and marked hypertrophic osteo $\rightarrow$ arthropathy of all long bones (Figs 3 to 6). There was no radiological evidence of joint involvement.

After consultation with the thoracic surgeon, it wa. decided that treatment should be in the first place conservative, and he was admitted for vigorous pos tural drainage, breathing exercises, and antibioticser Sputum culture grew a Haemophilus species sensitive to streptomycin, tetracycline, and chloramphenicol ${ }_{\odot 0}^{+}$ but the urine showed no abnormality. He was put onf chloramphenicol, but continued to run a low-grade pyrexia.

On 19 November 1968 bronchoscopy with bron chial lavage was performed. Following this his tem $<$ perature became swinging in nature and he coughecto up copious amounts of purulent sputum. On 228 November he fell out of bed and was found to have grossly increased tendon reflexes and a sustaineco right ankle clonus; a skull radiograph was normals On the following day he developed severe pain

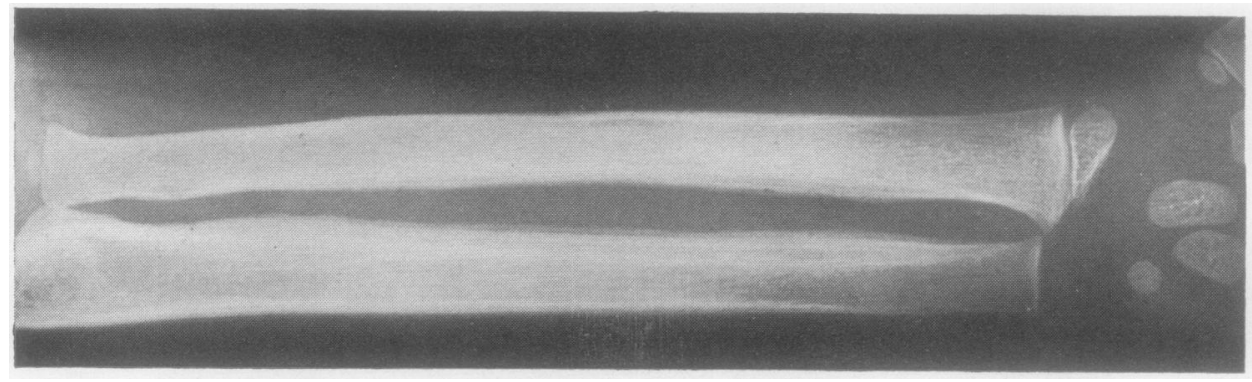

FIG. 5

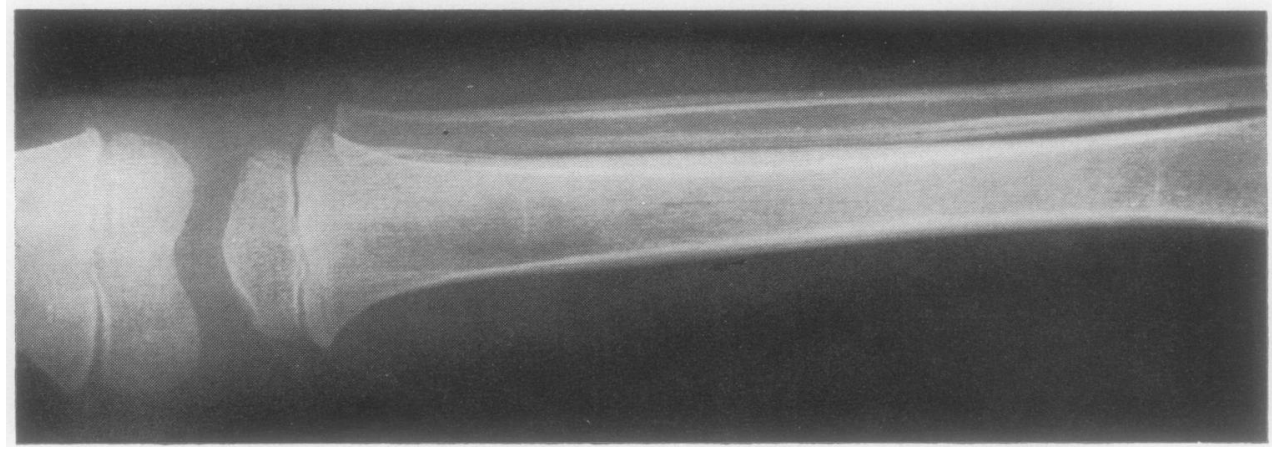

FIG. 6

FIGS 5 and 6. Periosteal hypertrophy of radius and ulna and tibia and fibula at the time of presentation. 
tenderness, and swelling of both forearms. He became extremely restless, and only after sedation with a combination of amytal, $100 \mathrm{mg}$., and chloral hydrate, 600 mg., 6-hourly did he settle in bed. Radiographs at this time showed a marked increase in the extent of the hypertrophic osteoarthropathy (Fig. 7). Blood and gas analysis results were:

$\mathrm{Po}_{2}=89 \mathrm{~mm} . \mathrm{Hg} ; \mathrm{PCO}_{2}=35 \mathrm{~mm} . \mathrm{Hg} ; p \mathrm{H}=7 \cdot 46$

On account of his persistent pyrexia, antibiotic treatment was changed from chloramphenicol to streptomycin. Blood cultures taken at this time grew a Staphylococcus pyogenes sensitive to penicillin, tetracycline, and chloramphenicol. He was bronchoscoped again on 3 December, but this examination revealed no new information and was followed by an extremely stormy period. Consciousness was not fully regained for 48 hours. He became severely anaemic due to repeated massive haemoptyses, his haemoglobin falling at one time to $3 \mathrm{~g} . / 100 \mathrm{ml}$. and in the next 10 days he was transfused with 8 pints of blood.

It became obvious that direct surgical intervention was the only course available. At operation on 14 December 1968 a tracheostomy was performed pro- phylactically during the operation to provide better access for ventilation and sucking out. The right lower lobe was found to be completely ballooned out with extravasated blood (which had filled the abscess cavity) from a ruptured pulmonary artery, and a right lower lobectomy was done. Histology of the resected specimen showed chronic inflammation and a plasma cell pneumonitis; there was no evidence of malignancy or tuberculosis.

His post-operative recovery was speedy and uneventful, apart from moderate elevation of blood pressure (140/95), which lasted for about 48 hours. As soon as he regained consciousness it was noticed that he was much more co-operative and less restless. By the fourth post-operative day, the swelling and tenderness of the forearms had disappeared, and the finger and toe clubbing was subsiding. The tracheostomy tube was removed on the 16 th post-operative day. He was discharged well on 7 January 1969.

Repeat chest radiographs three and a half months after lobectomy showed normal lung fields; there was also evidence of a considerable decrease in the sub-periosteal reaction of the long bones (Figs 8 and

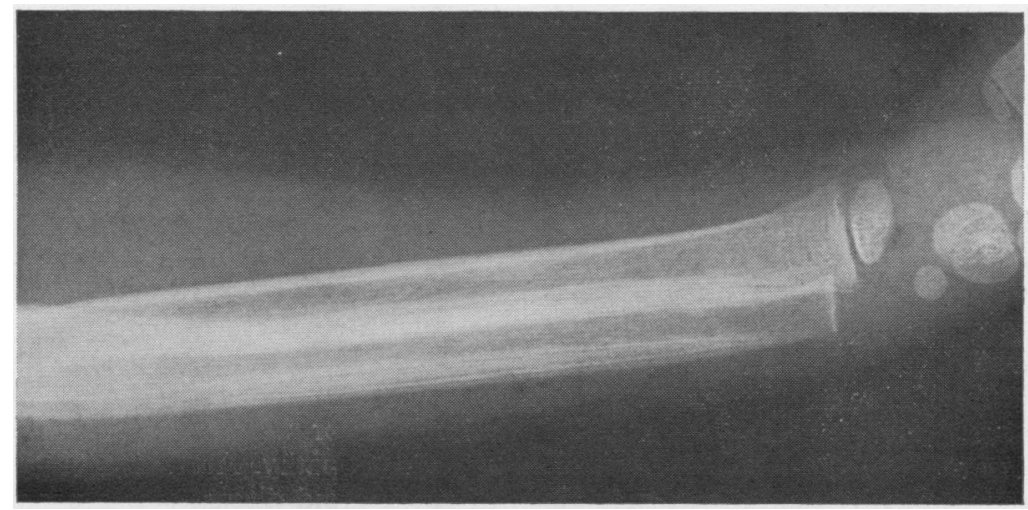

FIG. 7. Increase in periosteal hypertrophy of radius and ulna during exacerbation of illness immediately prior to operation.

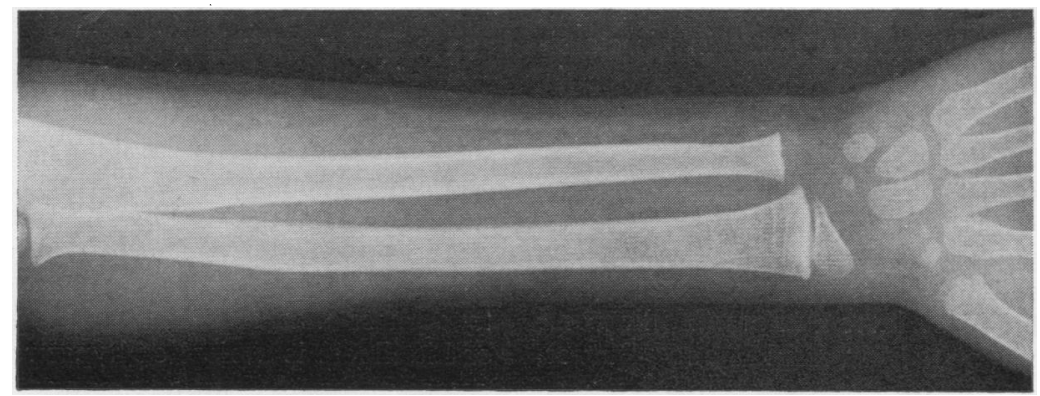

FIG. 8. Almost complete resolution of the periosteal hypertrophy of both radius and ulna 10 months after operation. 


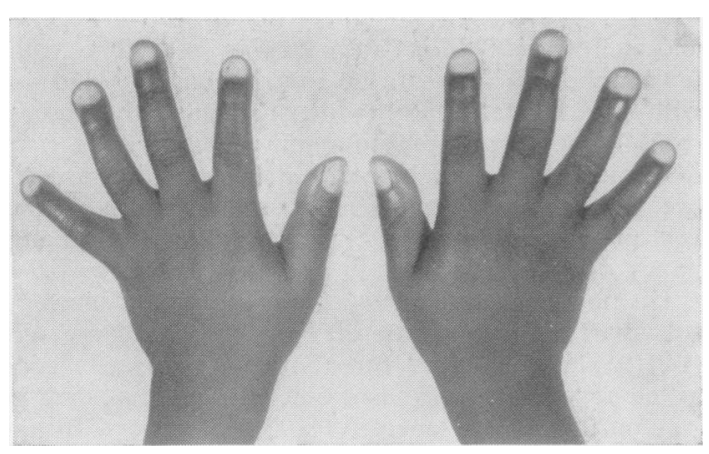

FIG. 9. Virtually normal hands 10 months after operation.

9), and clubbing of the digits had largely cleared. Blood gas studies at this time showed no significant change from those done prior to operation. Central nervous system signs had returned to normal and the child was again quiet and co-operative. He could now not only fight but actually beat his younger brother in play.

\section{DISCUSSION}

Cavanaugh's review in 1965 of the world literature on paediatric cases of hypertrophic pulmonary osteoarthropathy showed that out of the 39 patients, 25 were primarily due to chronic lung conditions ; 7 of these were tuberculous. Cyanotic heart lesions accounted for 6 patients, biliary cirrhosis, including one case of biliary atresia, for 3 , and 3 other cases had chest diseases, 1 malignant and 2 undetermined. There was a scatter of Negro and Caucasian children. With the decrease of chronic inflammatory lung disease in developed countries the rare complication of hypertrophic pulmonary osteoarthropathy is hardly seen in children. However, in countries such as Nigeria, where easy access to medical facilities is limited to a few areas, these cases still occur.

The exact pathogenesis of the condition is not known. Bamberger (1889) and Marie (1890) postulated a humoral agent ; Mendlowitz, in his classical experiments in 1941, found an increase in blood flow in clubbed fingers with a decrease in the pressure gradient across the same area. These findings, however, were not constant in his series, and readings were normal in his three cases of hypertrophic pulmonary osteoarthropathy. The increase in blood flow, whatever its mechanism, has been established by later authors (Holling and Brodey, 1961 ; Vogl, Blumenfeld, and Gutner, 1955).

More recently, a neural cause has been postulated. First described by Brea in 1949, both Flavell in 1956 and Diner in 1962 have confirmed the dramatic relief of symptoms following cervical or thoracic vagotomy in cases of unilateral lung disease. It has been well documented that the peripheral symptoms of hypertrophic pulmonary osteoarthropathy regress after resection of the causative lesion, although in some cases the structural abnormalities in the bones remain unaltered and may even progress. Symptoms do not recur if metastases of malignant lesions arise postoperatively (Diner, 1962; Vogl et al., 1955; Flavell, 1956). In considering the good results following vagotomy, one can postulate that the important part of the resection may well be the severance of the nerves supplying that part. The pleural surfaces have a more profuse nerve supply than the lung tissue itself ; this may account for the commoner association of hypertrophic pulmonary osteoarthropathy with lesions involving the pleural surface than with those in the centre of the lung tissue. It also explains why some conditions, such as dilated oesophagus, mediastinal tumours, and lesions of the ribs, can cause the same syndrome (Holling and Brodey, 1961).

The histopathology of the bone lesions as described by Holling and Brodey (1961) is one of an increase in connective tissue. Periosteal new bone is associated with a lymphocytic and plasma cell infiltrate of the surrounding connective tissue. When this involves the nail bed, clubbing occurs. In the dog this is preceded by an overgrowth of vascular connective tissue.

Clinically, patients may present either with the symptoms of the underlying disease or its complications, or with symptoms due entirely to the osteoarthropathy. Arthritis closely simulating that of rheumatoid disease was a prominent feature in nine of Cavanaugh's patients (Cavanaugh and Holman, 1965).

The patient discussed here is classical in many ways: his hypertrophic osteoarthropathy was associated with a suppurative lung lesion of four and a half years' duration. The absence of a history of joint or bone pain is probably not significant. In an environment where such symptoms are common, they tend to be ignored by both patients and their families until they become incapacitating. This is supported by the fact that his ankles were swollen at the time of presentation, as can be seen in Figure 2. The severe bone pain and tenderness that occurred during the exacerbation of his illness is typical of hypertrophic pulmonary osteoarthropathy in adults and in the familial variety, although it has been previously stated not to occur in childhood. $\mathrm{He}$ also displayed the classi- 
cal resolution of symptoms following surgery and the slower (but definite) disappearance of the structural abnormalities.

\section{REFERENCES}

Bamberger, E. (1889). Bronchiektasie. Wien. klin. Wschr., 2, 226 (Quoted by Cavanaugh and Holman, 1965).

Camp, J. D., and Scanlan, R. L. (1948). Chronic idiopathic hypertrophic osteo-arthropathy. Radiology, 50, 581

Cavanaugh, J. J. A., and Holman, G. H. (1965). Hypertrophic osteoarthropathy in childhood. J. Pediat., 66, 27.
Diner, W. C. (1962). Hypertrophic osteoarthropathy. J. Amer. med. Ass., 181, 555.

Flavell, G. (1956). Reversal of pulmonary hypertrophic osteoarthropathy by vagotomy. Lancet, 1, 260.

Holling, H. E., and Brodey, R. S. (1961). Pulmonary hypertrophic osteoarthropathy. J. Amer. med. Ass., 178, 977.

Marie, P. (1890). De l'ostéo-arthropathie hypertrophiante pneumique. Rev. Méd. (Paris), 10, 1 (Quoted by Camp and Scanlan, 1948).

Mendlowitz, M. (1941). Measurement of blood flow and blood pressure in clubbed fingers. J. clin. Invest., 20, 113.

Vogl, A., Blumenfeld, S., and Gutner, L. B. (1955). Diagnostic significance of pulmonary hypertrophic osteoarthropathy. Amer. J. Med., 18, 51 . 